\title{
Obturador palatino: confecção de uma prótese não convencional - relato de caso
}

\author{
Palatal obturator: confection of an unconventional prosthesis - \\ case report
}

\author{
Lisiane Aguiar* \\ André Roberto Mozzini** \\ Elder Lersch ${ }^{* * *}$ \\ Ferdinando De Conto ${ }^{* * * *}$
}

\begin{abstract}
Introdução: defeitos maxilares podem ser criados em decorrência de tratamentos cirúrgicos para neoplasias benignas ou malignas. Reabilitação com prótese obturadora é uma opção complementar de tratamento em casos em que procedimentos cirúrgicos são contraindicados. O tamanho e a localização do defeito influenciam no grau de dificuldade de reabilitação. Relato do caso: este relato clínico descreve a reabilitação protética de paciente submetido à maxilectomia parcial bilateral para tratamento de adenocarcinoma de palato que necessitou de procedimento não convencional para estabilização da prótese a partir de confecção de dispositivo tipo mola. Considerações finais: a prótese obturadora é usada para restaurar a função mastigatória e melhorar a fala, deglutição e estética desses pacientes.
\end{abstract}

Palavras-chave: Prótese maxilofacial. Obturadores palatinos. Reabilitação bucal.

\section{Introdução}

Defeitos maxilares, em sua maioria, originam-se devido às cirurgias para remoção de tumores, por vezes malignos, tais como os carcinomas; porém, defeitos congênitos, doenças infecciosas e os mais diferentes tipos de traumas também levam à necessidade de remoção parcial ou total da maxila. A reabilitação de tais pacientes é um desafio a ser enfrentado pelos profissionais envolvidos ${ }^{1}$.

Os carcinomas de palato são raros, principalmente pelo seu sítio anatômico, e quase sempre estão associados a hábitos nocivos, traumas ou doenças infectocontagiosas. O Brasil detém uma das mais altas taxas desse tipo de câncer no mundo. Apesar de esse tipo de câncer ter predileção por homens acima dos 40 anos, estudos mostram que o número de mulheres acometidas vem aumentando devido a mudanças de hábitos, registrando-se que, de todos os fatores a que se atribui a contribuição da etiologia do câncer bucal, o tabagismo e o etilismo, quando combinados, potencializam os riscos de desenvolver lesões cancerizáveis ${ }^{2,3}$.

Cirurgiã-Dentista graduada na Faculdade de Odontologia, Universidade de Passo Fundo, Passo Fundo, RS, Brasil.

Cirurgião de Cabeça e Pescoço, Faculdade de Medicina, Universidade de Passo Fundo; Hospital São Vicente de Paulo, Passo Fundo, RS, Brasil.

*** Médico Patologista; Hospital São Vicente de Paulo, Passo Fundo, RS, Brasil.

***** Cirurgião Bucomaxilofacial, Faculdade de Odontologia, Universidade de Passo Fundo; Hospital São Vicente de Paulo, Passo Fundo, RS, Brasil. 
Apesar de haver os mais variados tipos histopatológicos de tumores malignos, desde os mesenquimais até os de glândulas salivares menores, o tumor mais frequente na região de cabeça e pescoço é o carcinoma epidermoide em seus diferentes graus de diferenciação. Geralmente agressivo, leva em pouco tempo a grandes destruições teciduais, sendo necessárias cirurgias extensas para sua total remoção, obrigando profissional e paciente a buscarem alternativas de reconstrução da área atingida. No caso específico da região do palato, a necessidade de remoção de neoplasias quase sempre gera uma comunicação bucossinusal, que precisa ser interrompida ${ }^{1,4}$.

O tratamento primário do carcinoma de palato é cirúrgico, e a técnica é definida de acordo com o sítio e o tamanho da lesão. Comumente, opta-se por uma ressecção da lesão com área de segurança, ou, em casos mais graves, uma maxilectomia total ou parcial, uni ou bilateral, dependendo da localização e extensão da área atingida. A técnica pode ser classificada em três tipos: no tipo 1, há preservação do assoalho da órbita; no tipo 2 , há perda de suporte orbitário; e no tipo 3, ocorre a exenteração da órbita. A técnica cirúrgica leva sempre à necessidade de uma reconstrução da área atingida, não apenas no sentido funcional, devolvendo ao paciente sua função mastigatória e fonética, como também no sentido de auxiliar no processo de reabilitação emocional ${ }^{1}$. A reabilitação protética das perdas maxilares divide-se em três fases: cirúrgica, provisória e reabilitadora, cada qual com suas características, indicações e importâncias ${ }^{5}$.

A maxilectomia, seja ela parcial ou total, sempre acarreta deficiência nos mais diferentes graus de funcionalidade da cavidade oral. Comunicações bucossinusais são frequentes e precisam ser corrigidas, evitando, assim, que haja refluxo nasal e que a voz possa ser emitida sem interferências ${ }^{6}$.

A alternativa para esses casos é a utilização de um obturador palatino, que consiste em uma prótese parcial removível que substitui a área removida do tumor, tendo como função obliterar a comunicação bucossinusal, possibilitando que as funções orais prejudicadas sejam restabelecidas, além de dar suporte à face, permitindo que seu contorno volte à normalidade. Por meio do uso de obturador palatino, é possível uma reabilitação quase imediata das funções orais, bem como um controle da doença, visto que os obturadores são de fácil remoção, o que possibilita o controle de possíveis recidivas, além de auxiliar na recuperação física e psicológica do paciente $^{7}$.

O objetivo deste trabalho é relatar o caso clínico de uma paciente submetida a maxilectomia parcial para tratamento de adenocarcinoma de palato.

\section{Relato de caso}

Paciente do gênero feminino, 74 anos, procurou atendimento odontológico para reabilitação dentária após ser submetida a maxilectomia parcial para remoção de adenocarcinoma de palato (Figuras 1 e 2). O histórico revelou que a paciente havia sido submetida, há cerca de 12 meses, a tratamento cirúrgico, acompanhada por cirurgião oncológico. Não foi realizada avaliação odontológica prévia ao tratamento.

O tratamento para a lesão maligna constituiu-se de ressecção cirúrgica da maxila seguida de 39 sessões de radioterapia com dose total de 50 Gy. Durante esse período, a principal queixa da paciente - que foi acometida de severa perda de peso (14 quilos), devido à grande comunicação buconasal referia-se à dificuldade de alimentação, tendo se alimentado por sonda nasogástrica.

Após esse período, procurou o cirurgião-dentista para tentativa de confecção de prótese dentária, sendo, então, encaminhada para o serviço especializado para confecção de prótese dentária obturadora.

Durante o exame clínico e radiográfico (Figuras 3 e 4), foi constatada importante doença periodontal nos dentes remanescentes. Estes, porém, não puderam ser extraídos, em razão de a paciente ter sido submetida a tratamento radioterápico, o qual, associado a extrações dentárias, pode ocasionar sérias complicações, culminando em osteorradionecrose.

A paciente foi, então, encaminhada para dar início à confecção da prótese obturadora palatina, técnica que consiste em uma moldagem preliminar cuidadosa com alginato. Após moldagem e obtenção dos modelos de estudo, a prótese obturadora palatina convencional foi desenhada e confeccionada com resina acrílica, restabelecendo a dimensão vertical da paciente.

Após a instalação da prótese (Figuras 5 e 6), pôde-se observar uma imediata melhora na fonação, na deglutição e, logo, no bem-estar da paciente, a qual, porém, relatou uma importante queixa em relação à falta de sustentabilidade da prótese, mencionando que esta se deslocava com o movimento da abertura de boca. Em vista disso, optou-se por confeccionar um dispositivo metálico para que a prótese se mantivesse suspensa e estável. Tal dispositivo, instalado entre a prótese superior e a prótese parcial removível (PPR) inferior convencional, era removível, permitindo que a paciente o retirasse para facilitar a higienização da prótese (Figuras 7 e 8).

A paciente fez uso da prótese obturadora palatina por cerca de dois anos, até vir a falecer devido à evolução da doença metastática. 


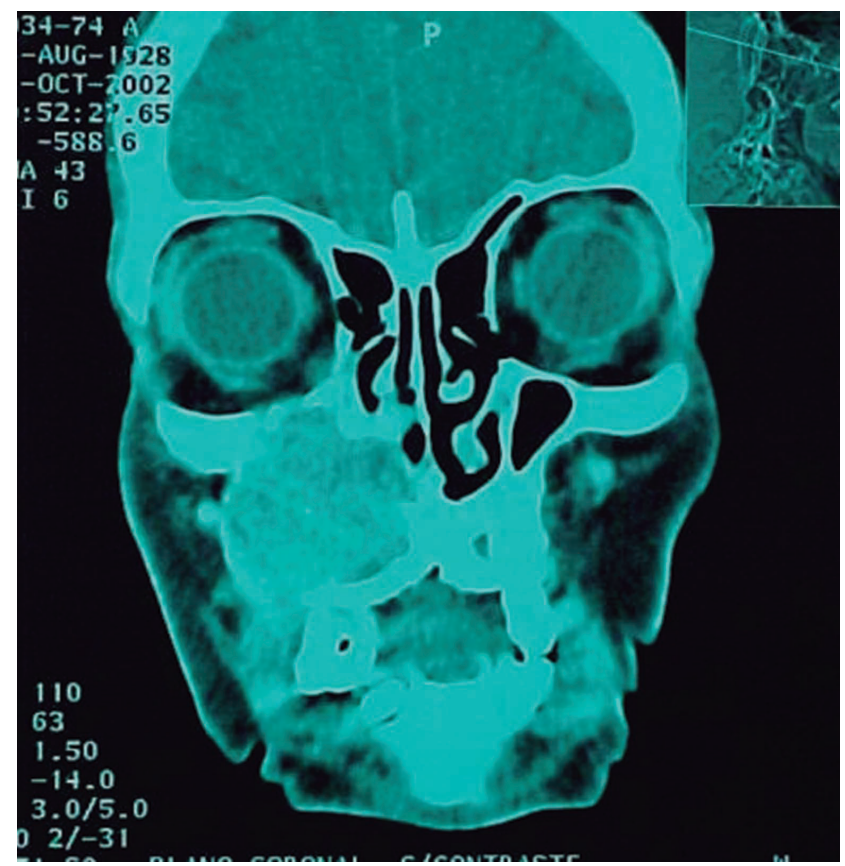

Figura 1- Massa expansiva com densidade de partes moles e impregnação heterogênea pelo meio de contraste, invadindo e ocupando o seio maxilar direito, onde se observa remodelamento e destruição das paredes ósseas

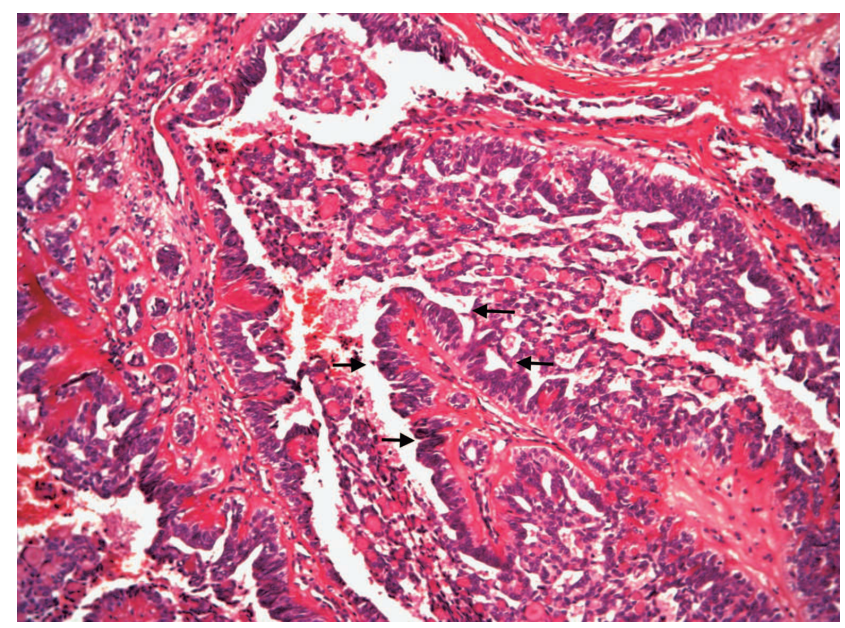

Figura 2 - Aspecto histopatológico compatível com adenocarcinoma moderadamente diferenciado; papilífero evidenciando diversas estruturas glandulares; setas evidenciam a formação papilar

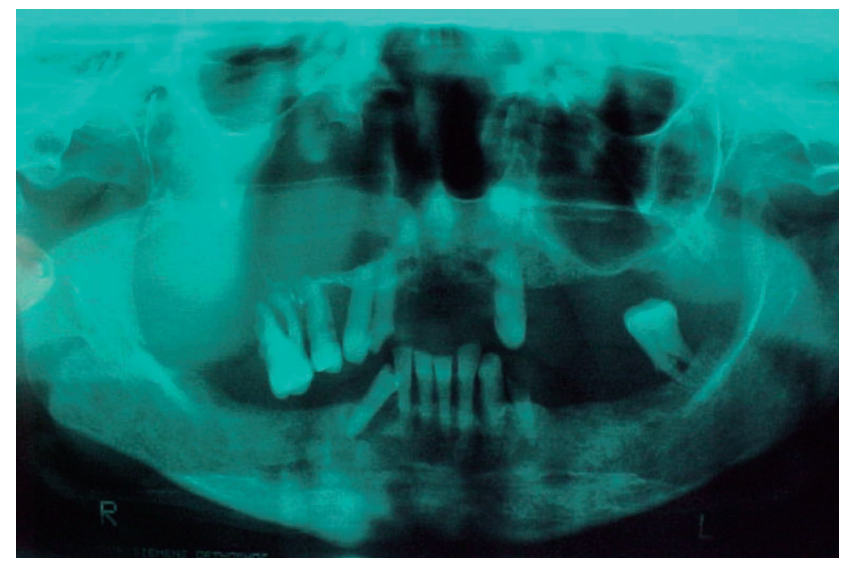

Figura 3 - Radiografia panorâmica evidenciando extensa lesão destrutiva na maxila do lado direito, além de estado periodontal ruim dos outros dentes da paciente

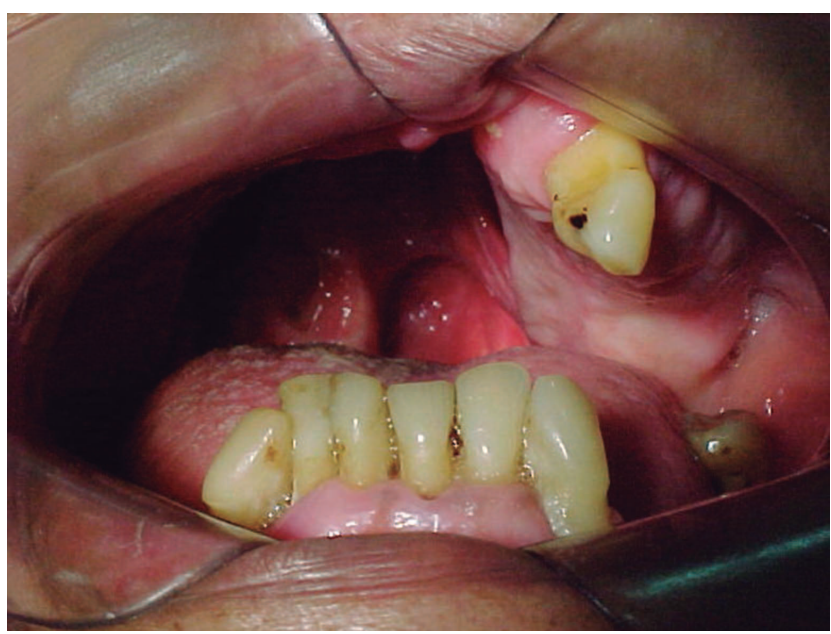

Figura 4 - Aspecto intraoral 12 meses após a maxilectomia direita. Paciente ainda apresentava trismo, que dificultava o exame clínico

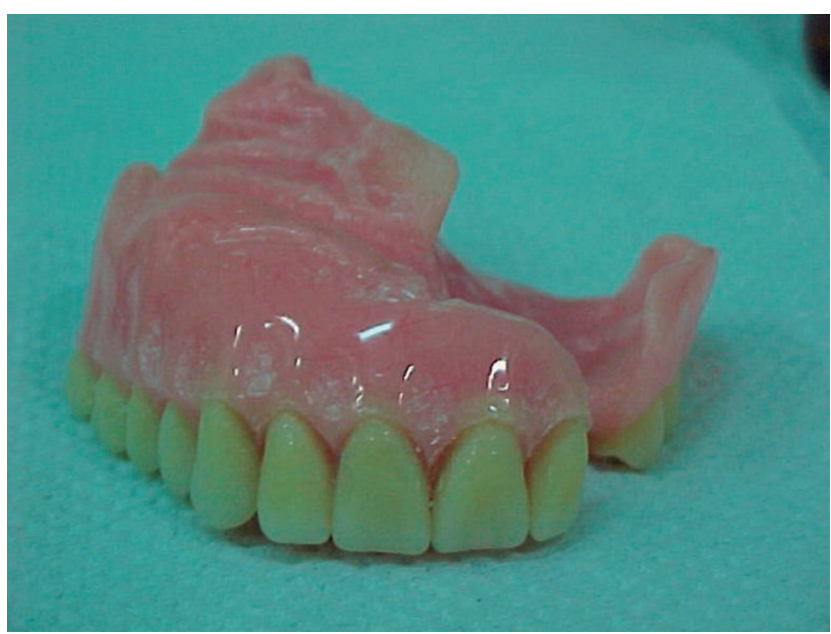

Figura 5 - Vista frontal da prótese obturadora - observar o volume do material acrílico para preencher o lado direito da paciente

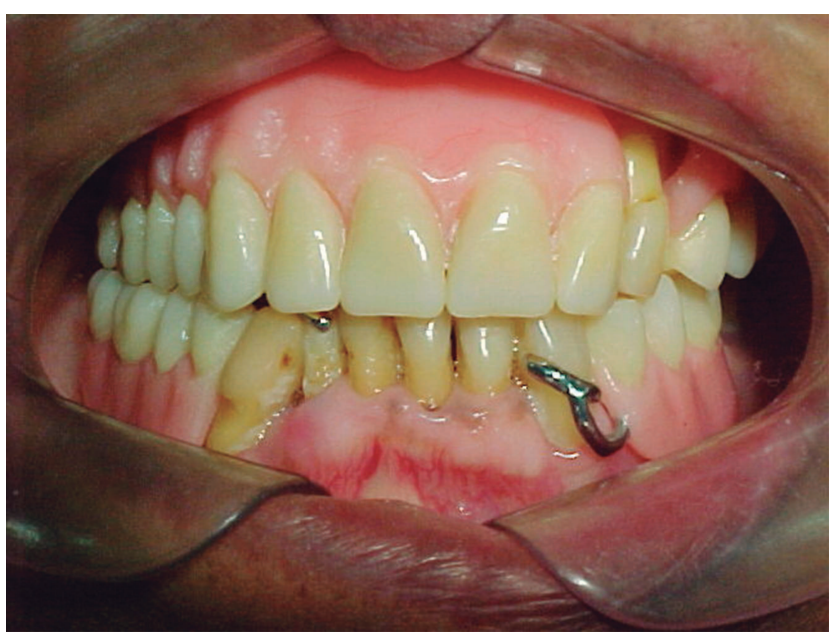

Figura 6 - Aspecto da oclusão final após a instalação da prótese obturadora superior e PPR inferior 


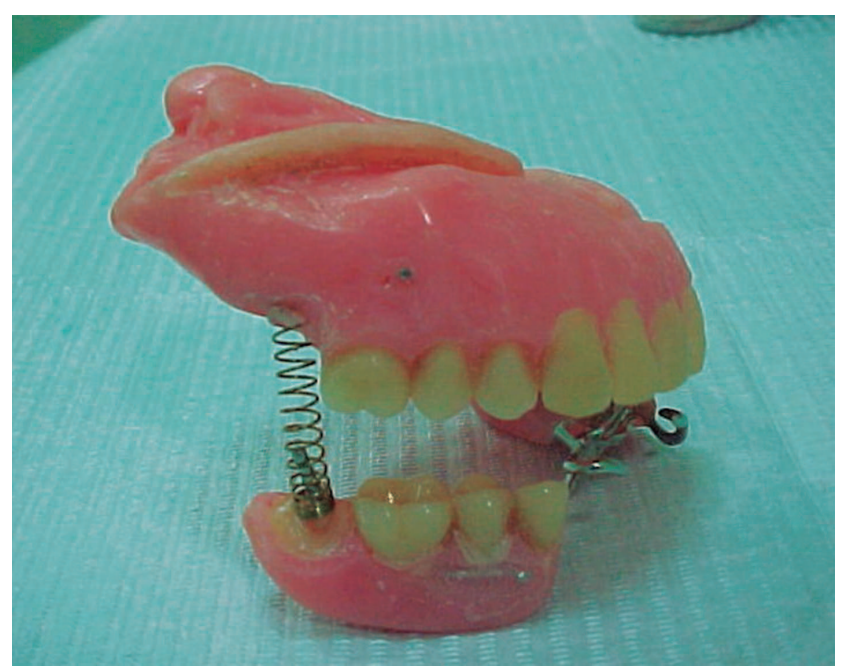

Figura 7- Vista lateral após a instalação do dispositivo tipo mola, utilizado para a estabilização da prótese superior

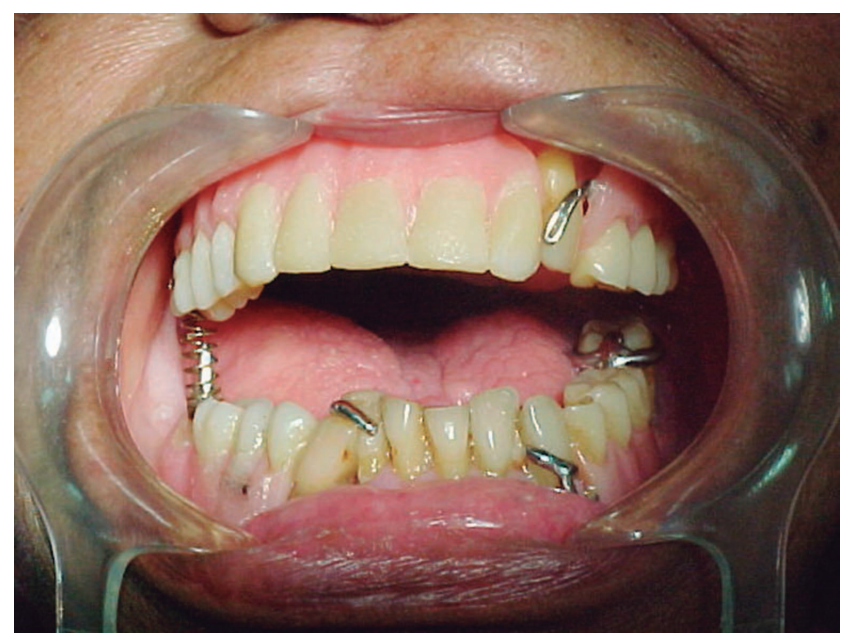

Figura 8 - Aspecto intrabucal após abertura de boca, comprovando a efetividade do dispositivo mola

\section{Discussão}

Dentre as principais complicações que podem ocorrer com as extrações dentárias durante o tratamento radioterápico, destaca-se a osteorradionecrose, que consiste em necrose isquêmica do osso devido à diminuição da vascularização tecidual. A necrose pode envolver o osso tanto superficial como profundamente, provocando fraturas patológicas dependendo da sua intensidade e extensão ${ }^{8}$.

As próteses obturadoras atuais podem ser confeccionadas com a utilização de dois materiais. $\mathrm{Na}$ região onde há necessidade de obliteração da comunicação buconasal, o uso de silicone é mais confortável para o paciente, podendo o restante da prótese ser confeccionado em resina acrílica para melhor durabilidade da mesma ${ }^{9}$.

Reabilitar pacientes acometidos de deformações faciais, sendo por traumas, tumores ou más-formações congênitas, é um grande desafio, o qual precisa ser suparado diante da imensa repercussão psicoló- gica imposta pelas deformações que o tratamento curativo impõe $\mathrm{e}^{5,10}$. Assim sendo, as próteses usadas como um recurso muitas vezes definitivo precisam ser confiáveis, funcionais e com uma estética agradável, para que a autoconfiança e a inserção social do paciente possam ser retomadas o mais breve possível. As próteses obturadoras enquadram-se nesses requisitos, sendo uma das opções mais aceitas para tratamentos imediatos, em razão de sua simplicidade, e, em diversos casos, utilizada como tratamento recuperador definitivo $0^{9,11}$.

A prótese obturadora tem suas indicações para pacientes que apresentem as mais variadas comunicações buconasais, sendo por remoção cirúrgica, destruição, ou ausência da maxila ${ }^{12}$. A confecção de um obturador palatino segue os princípios de uma prótese parcial removível, sendo uma técnica simples, rápida, eficaz, passível de adaptações e de fácil instalação, indicado para corrigir defeitos estéticos em pacientes que não podem optar pela cirurgia reconstrutiva ${ }^{9,13}$.

Para cada defeito maxilar, há uma possibilidade de confecção de um obturador palatino ${ }^{9}$. Dependendo da localização, da extensão, da presença ou ausência de estrutura de suporte e das condições gerais de saúde, é possível planejar e confeccionar uma prótese funcional direcionada às necessidades de cada paciente em particular. A remoção de parte ou da totalidade do osso maxilar cria problemas relacionados à estabilidade da futura prótese, sendo necessário, por vezes, confeccionar retenções adicionais para mantê-la em posição. Além disso, é preciso manter a prótese leve, para evitar que a ação da gravidade a desloque. Para isso, dispositivos removíveis podem ser instalados na prótese sem alterar sua conformação. Em casos mais complexos, implantes podem ser utilizados para dar estabilidade, retenção e suporte à prótese obturadora ${ }^{11}$.

No que se refere à deglutição e à fonação, os pacientes devem ser acompanhados por fonoaudiólogos e fisioterapeutas, cujo papel é auxiliar na realização de exercícios de deglutição, respiração e emissão de sons, fortalecendo a musculatura oral para que a adaptação da prótese possa ocorrer da melhor forma possível ${ }^{13}$.

São os pequenos detalhes que determinam o sucesso nesses casos, e planejamento multidisciplinar é fundamental ${ }^{10}$. Pacientes maxilectomizados perdem muito mais que apenas partes da região oral; ficam por vezes sem condições básicas de sobrevivência. Por envolver tratamentos que resultam em defeitos estéticos e funcionais importantes e visto que se trata de uma reabilitação complexa e demorada, exigindo uma visão mais ampla do tratamento que será imposto aos pacientes, o planejamento é essencial. Dessa forma, o tratamento reabilitador mediante a confecção de próteses bucomaxilofaciais, quando bem indicados e conduzidos, tem a capacidade de possibilitar a superação do trauma vivido pelo paciente, oportunizando-lhe uma melhor qualidade de vida, tornando-se este o objetivo principal desse tipo de tratamento ${ }^{13-16}$. 


\section{Considerações finais}

O cirurgião-dentista tem um papel fundamental na reabilitação biopsicossocial de pacientes maxilectomizados,pois, ao tratá-los com a utilizaçaõ das próteses bucimaxilofaciais, pode-lhes devolver uma feição saudável, diminuindo as sequelas deixadas pelo tratamento curativo e permitindo que a imagem social seja preservada. Pacientes maxilectomizados não precisam apenas de tratamentos curativos, mas também de soluções que permitam sua reintegração social e a retomada da sua vida cotidiana.

A busca por novas técnicas e novos materiais obturadores faz-se necessária visto que a adoção de hábitos nocivos pela população em geral está mais intensa e permite que doenças mutiladoras ocorram com frequência na cavidade oral. A orientação quanto aos malefícios do uso de tabaco e álcool, além de outros hábitos nocivos, deve ser adotada de forma sistemática nos consultórios e clínicas odontológicas, para que a função principal do cirurgião-dentista como a do profissional da saúde, ainda seja, predominantemente, a prevenção.

\section{Abstract}

Introduction: maxillary defects may be created by surgical treatment of benign or malignant neoplasms. Rehabilitation with obturator prosthesis is a complementary treatment option in cases where surgical procedures are contraindicated. The size and location of the defects influence the degree of difficulty for rehabilitation. Case report: this clinical report describes the prosthetic management of a patient subjected to bilateral partial maxillectomy for treatment of adenocarcinoma of the palate, which required an unconventional procedure for prosthesis stabilization by producing a spring device. Conclusion: the obturator prosthesis is used to restore masticatory function and improve speech, deglutition, and esthetics of these patients.

Keywords: Maxillofacial prosthesis. Palatal obturators. Oral rehabilitation.

\section{Referências}

1. Giovanini EG, Simonato LE, Castro EVFL de, Soubhia AMP, Castro AL de. Carcinoma mucoepidermóide de palato: descrição de caso clínico. RFO UPF. 2007; 12(1):61-4.

2. Dedivitis RA, França CM, Mafra ACB, Guimarães AV, Guimarães FT. Características clínicas-epidemiológicas no carcinoma espinocelular de boca e Orofaringe. Rev Bras otorrinolarin 2004; 70(1):35-40.

3. Brito AJP de, Fava AS, Makowiecky M, Ribas MH, Speck Filho J. Carcinoma espinocelular de palato mole. Rev Bras Cir Cabeça Pescoço 2008; 37(2):88-90.
4. Zini M, Moreschi E, Trento C L, Gottardo V D, Zardetto Jr $\mathrm{R}$, Aleixo T R de C. Carcinoma mucoepidermóide em palato: relato de caso. Rev Cir Traumatol Buco-Maxilo-Fac 2010;10(1):57-62.

5. Carvalho ACG de S, Castro Filho F M de, Sousa F B, Magro-Filho O, Romio K B, Nogueira R L M. Reabilitação bucal imediata após maxilectomia parcial: relato de caso. Rev Cir Traumatol Buco-Maxilo-Fac 2009; 9(2):33-8.

6. Pinto JHN, Pegoraro KMI. Evaluation of palatal prosthesis for the treatment of velopharyngeal dysfunction. J Appl Oral Sci 2003; 11(3):192-7.

7. Miracca RAA, Andrade Sobrinho J de, Gonçalves AJ. Reconstrução com prótese imediata pós maxilectomia. Rev Col Bras Cir 2007; 34(5):297-302.

8. Jacobson AS, Buchbinder D, Hu K, Urken ML. Paradigm shifts in the management of osteoradionecrosis of the mandible. Oral Oncol 2010; 46:795-801.

9. Tirelli G, Rizzo R, Biasotto M, Di Lenarda R, Argenti B, Gatto A, et al. Obturator prostheses following palatal resection: clinical cases. Acta Otorhinolaryngol Ital 2010; 30(1):33-9.

10. Mintz A. "De cuerpos y almas": rehabilitación integral del paciente mutilado facial. Actas odontol 2007; 4(1):23-7.

11. Patil PG, Patil SP. Fabrication of a hollow obturator as a single unit for management of bilateral subtotal maxillectomy. J Prosthodont 2012; 21(3):194-9.

12. Goiato MC, Piovezan AP, Santos DM dos, Gennari Filho, Assunção WG. Fatores que levam à utilização de uma prótese obturadora. Rev Odontol Araçatuba 2006; 27(2):101-6.

13. Silva DP da, Almeida FCS de, Vaccarezza GF ,Brandão TB , Cazal C, Caroli A, et al. Reabilitação com obturador maxilar após cirurgia oncológica: relato de casos: Pesq Bras Odontoped Clin Integr 2004; 4(2):125-30.

14. Fong JH, Wu HT, Chio YW, Gonzalez AM, Huang CK, Chen YW, et al. Comprehensive rehabilitation after severe maxillofacial injury in a cleft patient. J Chin Med Assoc 2010; 73(10):543-8

15. Hatamleh MM, Haylock C, Watson J, Watts DC. Maxillofacial prosthetic rehabilitation in the UK: a survey of maxillofacial prosthetists' and technologists' attitudes and opinions. Int J Oral Maxillofac Surg 2010; 39(112):1186-92.

16. Velasquez-Cayón RT, Flores-Ruiz R, Torres-Lagares D, Gonzáles-Guerreiro S, Gonzáles-Padilha D, Gutiérrez-Perez YJL. Uso de obturadores em cirugía oral y maxilofacial. Presentación de cinco casos clínicos. Rev Esp Cirug Oral y Maxilofac 2011; 33(1):22-6.

\section{Endereço para correspondência}

Ferdinando De Conto

Campus I - BR 285 - Km 292

Bairro São José

Faculdade de Odontologia da UPF

99052-900 Passo Fundo - RS

Fone: (54) 33168402

E-mail: ferdi@upf.br

Recebido: 25/04/2013. Aceito: 28/06/2013 